\title{
Transient Inactivation of Perirhinal Cortex Disrupts Encoding, Retrieval, and Consolidation of Object Recognition Memory
}

\author{
Boyer D. Winters and Timothy J. Bussey \\ Department of Experimental Psychology, University of Cambridge, Cambridge CB2 3EB, United Kingdom
}

\begin{abstract}
Damage to perirhinal cortex $(\mathrm{PRh})$ impairs object recognition memory in humans, monkeys, and rats when tested in tasks such as delayed nonmatching to sample, visual paired comparison, and its rodent analog, the spontaneous object recognition task. In the present study, we have capitalized on the discrete one-trial nature of the spontaneous object recognition task to investigate the role of PRh in several distinct stages of object recognition memory. In a series of experiments, transient inactivation of PRh was accomplished with bilateral infusions of lidocaine directly into PRh immediately before the sample phase (encoding), immediately before the choice phase (retrieval), or within the retention delay after the sample phase (storage-consolidation). Compared with performance on trials in which they received saline infusions, rats were significantly impaired when lidocaine was infused before the sample phase, regardless of the length of the retention delay. Similarly, delay-independent deficits were observed after immediate pre-choice infusions of lidocaine. Finally, PRh inactivation immediately and $20 \mathrm{~min}$ after the sample phase, but not 40, 60, or $80 \mathrm{~min}$ after, also disrupted subsequent object recognition when the retention delay was sufficiently long to ensure the dissipation of the actions of lidocaine during the choice phase. The effects of pre-sample and pre-choice inactivation indicate involvement of PRh in encoding and retrieval stages of object recognition, and the time course of post-sample inactivation effects suggests a role for PRh in the maintenance of the object trace during memory consolidation.
\end{abstract}

Key words: acquisition; lidocaine; object discrimination; rat; reversible lesion; storage

\section{Introduction}

Object recognition tasks such as delayed nonmatching to sample (DNMS), visual paired comparison, and its rodent analog, the spontaneous object recognition (SOR) task (Ennaceur and Delacour, 1988), have played an indispensable role in our understanding of the neurobiological basis of memory, finding application in imaging, neuropsychological, pharmacological, lesion, and genetic studies of learning and memory (Mishkin, 1978; ZolaMorgan and Squire, 1985; Zola-Morgan et al., 1986; McKee and Squire, 1993; Bartolini et al., 1996; Ennaceur et al., 1996; Buffalo et al., 1998; Elliott and Dolan, 1998; Murray and Mishkin, 1998; Bussey et al., 1999; Pascalis and Bachevalier, 1999; Tang et al., 1999; Clark et al., 2000; Manns et al., 2000; Pitsikas et al., 2001; Bozon et al., 2003; Duzel et al., 2003; Nemanic et al., 2004; Richmond et al., 2004). There is now strong evidence that one of the most important structures in visual recognition memory is the perirhinal cortex (PRh) (Murray and Bussey, 1999). Damage to

Received Sept. 15, 2004; revised Nov. 5, 2004; accepted Nov. 14, 2004.

This work was supported by a Wellcome Trust Project grant to T.J.B. and Dr. L.M. Saksida. B.D.W. was additionally supported by a fellowship under the Merck Sharp and Dohme Educational Sponsorship Program. This research was conducted under the auspices of the Cambridge Medical Research Council Centre for Behavioral and Clinical Neuroscience. We thank Simon Blosse for assistance with data collection for experiment $3 \mathrm{a}$.

Correspondence should be addressed to Dr. B. D. Winters, Department of Experimental Psychology, University of Cambridge, Downing Street, Cambridge CB2 3EB, UK. E-mail: bdw23@cam.ac.uk.

DOI:10.1523/JNEUROSCI.3827-04.2005

Copyright $\odot 2005$ Society for Neuroscience $\quad$ 0270-6474/05/250052-10\$15.00/0
PRh disrupts object recognition in humans (Buffalo et al., 1998), monkeys (Zola-Morgan et al., 1989; Gaffan, 1994), and rats (Mumby and Pinel, 1994; Ennaceur et al., 1996; Bussey et al., 1999, 2000; Winters et al., 2004). Indeed, lesions of PRh cause far greater disruption of object recognition memory in monkeys than damage to any other single structure in the medial temporal lobe (Meunier et al., 1993). Similarly, we have recently found, using a version of the SOR task modified to minimize possible spatial-contextual and locomotor confounds, no effect of lesions of the hippocampus in the face of substantial deficits after lesions of PRh [the opposite pattern of effects was obtained on a spatial task (Winters et al., 2004; Forwood et al., 2005)]. This double dissociation of function further emphasizes the critical role played by $\mathrm{PRh}$ in object recognition memory.

The foregoing lesion studies have involved permanent $\mathrm{PRh}$ damage. Such studies have proven invaluable in elucidating the anatomical locus of recognition memory. There is certain information, however, that permanent lesions cannot provide, such as for what stage of memory (encoding, consolidation, and/or retrieval) a particular brain region is necessary. In the present study, we have addressed this question by capitalizing on the discrete one-trial nature of the SOR paradigm to test the effects of transient lidocaine-induced PRh inactivation during sample presentation (encoding), during the choice phase (retrieval), and during the retention interval (consolidation). It was found that PRh is critical for all stages of visual recognition memory. 


\section{Materials and Methods}

Subjects

The subjects were 110 adult male Lister Hooded rats (Harlan Olac, Bicester, UK), weighing 270-320 gm before surgery and housed in pairs in a room with a $12 \mathrm{hr}$ light/dark cycle (lights on at 7:00 P.M.). All behavioral testing was conducted during the dark phase of the cycle. During testing, rats were fed $\sim 15$ gm of laboratory chow after daily behavioral sessions to maintain weights at $85-90 \%$ of free-feeding body weight. Water was available ad libitum throughout the experiment. All experimentation was conducted in accordance with the United Kingdom Animals (Scientific Procedures) Act, 1986.

\section{Surgery}

For each experiment, all rats were implanted bilaterally with 22 gauge indwelling guide cannulas according to the following procedure. Before surgery, all animals were deeply anesthetized by intraperitoneal injection $(60 \mathrm{mg} / \mathrm{kg}$ ) of sodium pentobarbital (Sagatal; Rhône Mérieux, Essex, UK) and placed in a stereotaxic frame (David Kopf Instruments, Tujunga, CA) with the incisor bar set at $-3.2 \mathrm{~mm}$. The scalp was cut and retracted to expose the skull. Holes were drilled, and the guide cannulas were implanted according to the following coordinates, measured relative to the skull at bregma (Paxinos and Watson, 1997): anteroposterior, $-5.5 \mathrm{~mm}$; lateral, $\pm 6.6 \mathrm{~mm}$; dorsoventral, $-6.5 \mathrm{~mm}$. The cannulas were secured to the skull using four jeweler screws and dental acrylic. Obdurators cut to extend $1.1 \mathrm{~mm}$ beyond the tip of the guide cannulas and with an outer diameter of $0.36 \mathrm{~mm}$ were inserted into the guides and remained there except during infusions. At the completion of each surgery, the skin was sutured, and an antibiotic powder (Acramide; Dales Pharmaceuticals, Skipton, UK) was applied. Animals were allowed to recover for at least $7 \mathrm{~d}$ before the beginning of behavioral testing.

\section{Histology}

After behavioral testing, rats were anesthetized by intraperitoneal injection of $2 \mathrm{ml}$ of Euthatal (Rhône Mérieux) and perfused transcardially with $100 \mathrm{ml}$ of PBS, pH 7.4, followed by $250 \mathrm{ml}$ of $4 \%$ paraformaldehyde (PFA), pH 7.4. The brains were removed, postfixed in $4 \% \mathrm{PFA}$ at $4^{\circ} \mathrm{C}$ for $24 \mathrm{hr}$. and then immersed in $25 \%$ sucrose in PBS until they sank. Coronal sections $(60 \mu \mathrm{m})$ were cut on a freezing microtome through the extent of $\mathrm{PRh}$, and every fifth section was mounted on a gelatin-coated glass slide and stained with cresyl violet. Slides were examined under a light microscope to verify the cannula placements.

\section{Infusion procedure}

For all experiments, rats received bilateral infusions of either physiological saline ( $0.9 \%$ sodium chloride, $\mathrm{pH} 7.0$; Aquapharm, Animalcare Limited, York, UK) or lidocaine hydrochloride ( $2 \%$ in physiological saline; Sigma, Poole, UK) on a given trial. For all conditions, the basic procedure was as follows. All infusions took place in a preparation room separate from the behavioral testing area. Animals were gently restrained by the experimenter throughout the infusion process. The obdurators were removed, and the 28 gauge infusion cannulas, which were cut to extend 1 $\mathrm{mm}$ beyond the tip of the guides, were inserted into the guides. Bilateral infusions were conducted simultaneously using two $1 \mu$ l Hamilton syringes, which were connected to the infusion cannulas by propylene tubing. The syringes were driven by a Harvard Apparatus precision syringe pump, which delivered $0.5 \mu$ l to each hemisphere over $1 \mathrm{~min}$. The infusion cannulas were left in place for an addition $1.5 \mathrm{~min}$ to allow for diffusion of the infusate. The infusion cannulas were then removed, and the obdurators were replaced before the next phase of the procedure (see below for specific experiment parameters). For all experiments, in each of the two habituation sessions before the beginning of behavioral testing, rats experienced a "mock" infusion identical in all aspects to the procedure described above, except that the injection cannulas contained no liquid. This was done to habituate the animals to the general aspects of the infusion procedure, including insertion of the infusion cannulas and the sound of the pump.

\section{Autoradiography of lidocaine diffusion}

The distribution of $0.5 \mu \mathrm{l}$ of $2 \%\left[{ }^{14} \mathrm{C}\right]$-lidocaine hydrochloride $(26.95$ $\mathrm{mCi} / \mathrm{mmol}$ ) (PerkinElmer Life Sciences, Wellesley, MA) was assessed in two subjects not run during the behavioral sessions. These rats underwent the same surgical procedures as those in the behavioral studies and were habituated with two mock infusion days before the actual infusion of $\left[{ }^{14} \mathrm{C}\right]$-lidocaine hydrochloride. Five minutes after an injection according to the parameters described above, the rats were overdosed with an intraperitoneal injection of $2 \mathrm{ml}$ of Euthatal, and the brains were rapidly removed and frozen on dry ice. The brains were embedded and sectioned $(20 \mu \mathrm{m})$ in a $-18^{\circ} \mathrm{C}$ cryostat, and every 10 th section was mounted onto a gelatin-coated glass slide and stored at $-18^{\circ} \mathrm{C}$ for $72 \mathrm{hr}$. The slides were then placed in a cassette and apposed to Eastman Kodak (Rochester, NY) Biomax MR single emulsion film for $72 \mathrm{hr}$ at room temperature. The film was then developed for $5 \mathrm{~min}$ to visualize the extent of diffusion of the lidocaine. The brain sections were subsequently stained with cresyl violet to verify the cannula tip locations.

\section{Spontaneous object recognition}

Apparatus. Spontaneous object recognition was conducted in a Y-shaped apparatus, as described previously (Winters et al., 2004; Forwood et al., 2005). Briefly, the Y-shaped apparatus had high, homogeneous white walls constructed from Foamalux (Brett Martin, Lancashire, UK) to prevent the rat from looking out into the room and thereby maximize attention to the object stimuli. The apparatus was raised $30 \mathrm{~cm}$ from the floor with walls $40 \mathrm{~cm}$ high. Each arm was $27 \mathrm{~cm}$ in length and $10 \mathrm{~cm}$ wide. The start arm contained a guillotine door $18 \mathrm{~cm}$ from the rear of the arm. This provided a start box area within which the rat could be confined at the start of the sample and choice phases of a given trial. The floor and walls of the apparatus were wiped down with a dry paper towel between rats but otherwise were not cleaned during the experiment. A video camera was mounted above the apparatus to record all trials. Triplicate copies were obtained of the objects, which were made of glass, plastic, or metal. For any given trial, the objects in a pair were composed of the same material so that they could not be readily distinguished by olfactory cues. The height of the objects ranged from 5 to $20 \mathrm{~cm}$, and all objects were affixed to the floor of the apparatus with Blu Tack (Bostik, Stafford, UK) to prevent them from being displaced during a trial. As far as could be determined, the objects had no natural significance for the rats, and they had never been associated with a reinforcer.

General procedure. All rats were habituated in two consecutive daily sessions in which they were allowed to explore the empty Y-shaped apparatus for $5 \mathrm{~min}$. Before being placed in the apparatus, rats experienced a mock infusion procedure, as described above. After the mock infusion, the rats were taken from the preparation area to the testing room and placed in the start box; the guillotine door was then opened to allow the rat to explore the main area of the apparatus. When the rat exited the start box, the guillotine door was lowered to prevent reentry into this area of the apparatus. The experimenter did not begin timing the session until after the rat exited the start box. Testing began $24 \mathrm{hr}$ after the second habituation session. Rats were given a series of test trials (one per day), with a minimum interval of $24 \mathrm{hr}$ between trials. A different object pair was used for each trial for a given animal, and the order of exposure to object pairs as well as the designated sample and novel objects for each pair were counterbalanced within and across groups. The time spent exploring objects was assessed from video recordings of the sample and choice phases. Data were collected by scoring exploratory bouts using a personal computer running a program written in QuickBASIC 4.5. All trials were run with the experimenter blind to the drug treatment conditions.

Object recognition test. Each trial consisted of two phases. In the sample phase, two identical objects ( $\mathrm{A} 1$ and $\mathrm{A} 2$ ) were placed in the Y-shaped apparatus, one at the end of each exploration arm. The rat was placed in the start box with the guillotine door lowered. The guillotine door was then raised to allow the rat into the exploration area of the maze. When the rat exited the start box, the guillotine door was lowered to prevent reentry, and the sample phase began. The time spent exploring the two objects was scored by an experimenter viewing the rat on a video screen. The cumulative duration of exploratory bouts, the beginning and end of which were indicated by pressing a given key on the computer keyboard, was calculated by the computer program. Exploration of an object was defined as directing the nose to the object at a distance of $<2 \mathrm{~cm}$ and/or 
touching it with the nose. Turning around or sitting on the object was not considered exploratory behavior. The sample phase ended when the rat had explored the identical objects for a total of $25 \mathrm{sec}$.

At the end of the sample phase, the rat was removed from the Y-shaped apparatus for the duration of the retention delay. After the delay, the rat was placed back in the start box of the Y-shaped apparatus and released into the exploration area for the choice phase. The Y-shaped apparatus now contained an identical copy of the sample (familiar) object (A3) in one arm and a new object (B) in the other. The exploration arms in which the choice objects were placed were counterbalanced between rats and across trials. The rat was allowed to explore the objects for $2 \mathrm{~min}$, at the end of which it was removed and returned to its home cage. The time spent exploring the novel and familiar objects was recorded for the 2 min of the choice phase, but attention was focused on the first minute, during which object discrimination is typically greatest. We calculated a discrimination ratio, the proportion of total exploration time spent exploring the novel object (i.e., the difference in time spent exploring the novel and familiar objects divided by the total time spent exploring the objects), for the first minute of the choice phase on each object recognition trial. This measure takes into account individual differences in the total amount of exploration time.

Table 1 summarizes the details of each of the five experiments described in the following sections.

Experiment 1. Experiment 1 assessed the effects of intra-PRh infusions of lidocaine in three groups of rats. (1) The pre-sample group $(n=8)$ received infusions, as described above, immediately before the sample phase. Animals were placed in the Y-shaped apparatus $\sim 3$ min after the end of the infusion (disengagement of the pump). This should have provided enough time for the lidocaine to take effect, because previous studies suggest that it becomes active within $1 \mathrm{~min}$ of infusion (Boeijinga et al., 1993; Seamans and Phillips, 1994; Floresco et al., 1996). (2) The post-sample group $(n=8)$ received infusions immediately after the sample phase. After completion of the requisite $25 \mathrm{sec}$ of sample object exploration, the rat was removed from the apparatus and transported immediately to the infusion area for the start of intra-PRh infusions. (3) The pre-choice group $(n=8)$ received infusions immediately before the choice phase. The infusion procedure began with removal of the obdurators $\sim 4.5 \mathrm{~min}$ before the end of the retention delay. Animals were placed in the $\mathrm{Y}$-shaped apparatus for choice testing $\sim 3 \mathrm{~min}$ after the end of the infusion. The retention delay between the sample and choice phase was $20 \mathrm{~min}$ in experiment 1 . This should have allowed sufficient time for the effects of post-sample lidocaine infusions to dissipate before the choice phase, because previous studies suggest that the duration of activity of lidocaine is no longer than 10-15 min (Boeijinga et al., 1993; Seamans and Phillips, 1994). Animals were transferred to individual holding cages in a room adjacent to the testing room for the retention delay. All rats were run for a total of four trials, two with saline infusions and two with lidocaine infusions. The order of drug infusions was counterbalanced within and between groups.

Experiment 2a. Experiment 2a further assessed the effects of presample and pre-choice infusions of lidocaine by testing animals in the spontaneous object recognition task with relatively short $(5 \mathrm{~min})$ and long (180 $\mathrm{min}$ ) retention delays between the sample and choice phases. Two groups (pre-sample, $n=10$; pre-choice, $n=10$ ) were tested for eight trials, receiving saline infusions on half of the trials and lidocaine infusions on the other half. Half of the trials under each drug condition were run with a 5 min retention delay, and the other half involved a 180 min delay. Both the drug and delay orders were counterbalanced within and between the groups. All other parameters were identical to those described for experiment 1 .
Experiment $2 b$. Experiment $2 \mathrm{~b}$ followed on from experiment $2 \mathrm{a}$ by comparing the effects of pre-sample infusions of saline and lidocaine with three different retention delays: a minimum delay between sample and choice phases ("minimum"), $5 \mathrm{~min}$, and $180 \mathrm{~min}$. A single group of 11 rats was tested over 12 trials, receiving intra-PRh saline infusions on half of the trials and lidocaine on the other half. Each rat experienced two trials with each retention delay under each drug condition, and the orders of testing with drug and delay conditions were both counterbalanced. For the minimum and $5 \mathrm{~min}$ retention delays, rats were placed into a carrying box in the testing room for the duration of the delay. For the minimum condition, the delay lasted just long enough for the experimenter to change the objects for the choice phase; this procedure required a maximum of $30 \mathrm{sec}$. For the $180 \mathrm{~min}$ delay, rats were transferred to individual holding cages in a room adjacent to the testing room for the duration of the delay. Infusion parameters were the same as those for the pre-sample groups in experiments 1 and 2a.

Experiment 3a. Experiment 3a was designed to examine further the effects of post-sample infusions. The retention delay in this experiment was $60 \mathrm{~min}$. The effects of post-sample infusions were tested in three groups. (1) The immediate group $(n=9)$ received infusions of either saline or lidocaine immediately after the sample phase, as described for the post-sample group in experiment 1 . (2) The 20 min group $(n=9)$ received infusions $20 \mathrm{~min}$ after the end of the sample phase. At the end of the sample phase, these rats were transported to individual holding cages in an adjacent room until $20 \mathrm{~min}$ had passed. They were then given intra-PRh infusions of saline or lidocaine and returned to the holding cages for the remainder of the retention delay. (3) The $40 \min$ group $(n=$ 9) underwent the same procedure as the 20 min group, except that the infusions were delivered at $40 \mathrm{~min}$ after the end of the sample phase. All rats remained in the individual holding cages in the adjacent room throughout the duration of the retention delay when they were not receiving infusions. Each of the three groups was tested on four trials, two with saline and two with lidocaine, and the order of drug infusions was counterbalanced within and between groups.

Experiment 3b. Experiment $3 \mathrm{~b}$ was a replication and extension of experiment $3 \mathrm{a}$. To verify and extend the effects of post-sample infusions observed in experiment $3 a$, four groups of animals were tested with a retention delay of $120 \mathrm{~min}$. (1) The immediate group $(n=7)$ received infusions of either saline or lidocaine immediately after the sample phase, as described for the post-sample group in experiment 1. (2) The $20 \mathrm{~min}$ group $(n=7)$ underwent the same procedure as the 20 min group from experiment 3a. (3) The $60 \mathrm{~min}$ group $(n=7)$ underwent the same procedure as the $20 \mathrm{~min}$ group, except that the infusions were delivered at 60 min after the end of the sample phase. (4) The 80 min group $(n=7)$ 
received infusion at $80 \mathrm{~min}$ after the end of the sample phase. All rats remained in the individual holding cages in the adjacent room throughout the duration of the retention delay when they were not receiving infusions. Each group was tested on four trials, two with saline and two with lidocaine, and the order of drug infusions was counterbalanced within and between groups.

\section{Data analysis}

Group means of three measures taken from object recognition testing (duration of the sample phase, total exploration time in the choice phase, and the discrimination ratio) were analyzed for each experiment. Means from the sample duration and choice exploration data for experiments 1 , $2 \mathrm{a}, 3 \mathrm{a}$, and $3 \mathrm{~b}$ were submitted to two-way (group by drug) ANOVAs with repeated measures. For experiment $2 b$, the sample duration and choice exploration analyses were performed using paired-samples $t$ tests comparing data from the two drug conditions. Means for the discrimination ratio were submitted to two-way (group by drug: experiments $1,3 \mathrm{a}$, and 3 ; drug by delay: experiment $2 \mathrm{~b}$ ) or three-way (group by drug by delay: experiment 2a) ANOVAs with repeated measures. Significant interaction effects were further analyzed with separate paired-samples $t$ tests comparing the saline and lidocaine means within each group. All statistical analyses were conducted with a significance level of $\alpha=0.05$.

\section{Results}

Cannula placements

All rats included in the behavioral analyses had guide cannulas located bilaterally with injection needle tips terminating in PRh near the border between areas 35 and 36 within cortical layers 2-5 (Burwell, 2001). These placements were consistently located between 5.30 and $6.04 \mathrm{~mm}$ posterior to bregma, the approximate midsection of the rostral-caudal extent of PRh. Figure $1 a$ illustrates the placements of injection needle tips from the $20 \mathrm{~min}$ group in experiment 2a. This figure is representative of the distribution of cannula tips throughout all of the groups in all experiments discussed in the present study. Animals with placements centered outside of this region within PRh were excluded from behavioral analysis.

\section{Diffusion of lidocaine}

Both of the rats infused with $\left[{ }^{14} \mathrm{C}\right]$-lidocaine hydrochloride had cannula tip placements similar to those from the behavioral experiments (Fig. 1b). Qualitative analysis of the diffusion area of the radiolabeled lidocaine in the brains of these rats indicated a relatively discrete area of spread that included almost the entire rostral-caudal extent of PRh. Figure 2 illustrates the typical diffusion area within $\mathrm{PRh}$ in a representative section. In both animals, traces of radiolabeled lidocaine were observed in an area extending from $\sim 3.1 \mathrm{~mm}$ posterior to bregma (the rostral border of PRh) to $\sim 7.64 \mathrm{~mm}$ posterior to bregma. The radiolabel signal was stronger closer to the origin of the infusion but was also relatively high throughout the extent of PRh. Indeed, PRh was the only area consistently and bilaterally labeled throughout the diffusion area in both brains analyzed. There were, however, light traces within a very limited region of the ventral part of area TE closest to the cannula tip in one hemisphere of both brains. There was also a more consistent encroachment into the very dorsal parts of lateral entorhinal cortex unilaterally in both rats. Again, this was strongest near the origin of the infusion. There was no sign of diffusion into adjacent hippocampal regions.

\section{Experiment 1: stages of object recognition memory Histology}

Three rats, two from the post-sample group and one from the pre-choice group, were excluded from the final behavioral analyses for experiment 1 . In all three cases, infusion needle tracks
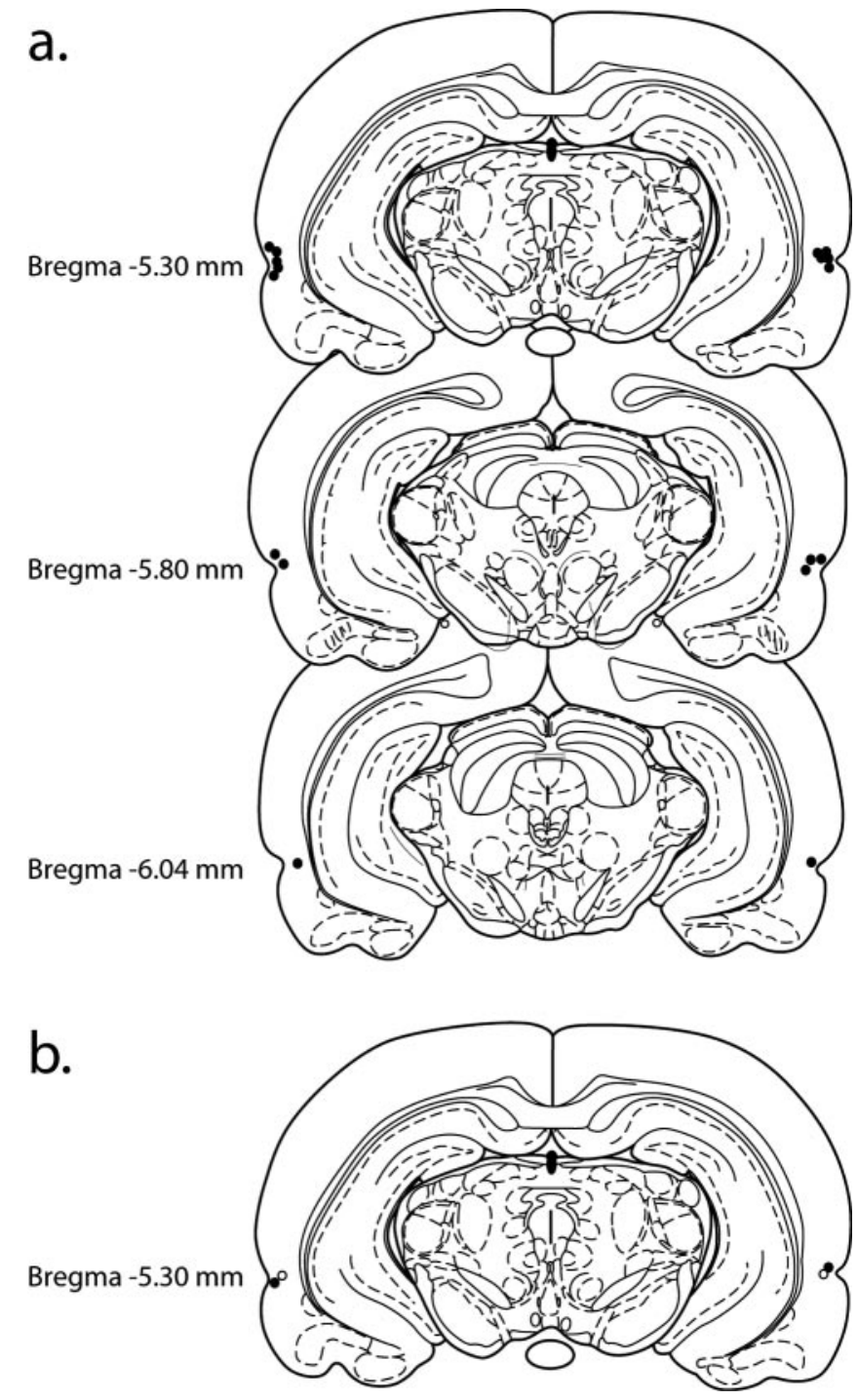

Figure 1. a, Schematic representation of the infusion needle tip placements from a typical group of animals (experiment $2 a, 20$ min group). These placements are representative of needle tip locations in all animals included in the behavioral analyses of the present study. Cannulas were consistently located between 5.30 and $6.04 \mathrm{~mm}$ posterior to bregma (Paxinos and Watson, 1997). Some needle tips are overlapping in the figure. $b$, Needle tip locations from the two rats infused with [ $\left.{ }^{14} \mathrm{C}\right]$-lidocaine hydrochloride to determine the extent of diffusion. Open circles are the locations of needle tips from the brain shown in Figure 2.

were judged to be too ventral, being centered in lateral entorhinal cortex rather than PRh. All animals included in the final analyses had infusion needle tips located as shown in Figure $1 a$.

\section{Spontaneous object recognition}

Duration of sample phase. The total time required to meet the sample object exploration criterion (25 sec cumulative) was analyzed, because a group difference at this stage of the trial might influence subsequent recognition performance. Analysis of the total time in the apparatus during the sample period revealed no significant difference between the groups $\left(F_{(2,18)}=1.71\right)$, no significant effect of drug $(F<1)$, and no group by drug interaction $(F<1)$. The mean sample phase duration $( \pm$ SEM) for each group under each drug condition was as follows: pre-sample, saline $=134.36 \pm 21.88 \mathrm{sec}$, lidocaine $=142.92 \pm 16.95 \mathrm{sec}$; post-sample, saline $=112.48 \pm 25.26 \mathrm{sec}$, lidocaine $=76.50 \pm$ 


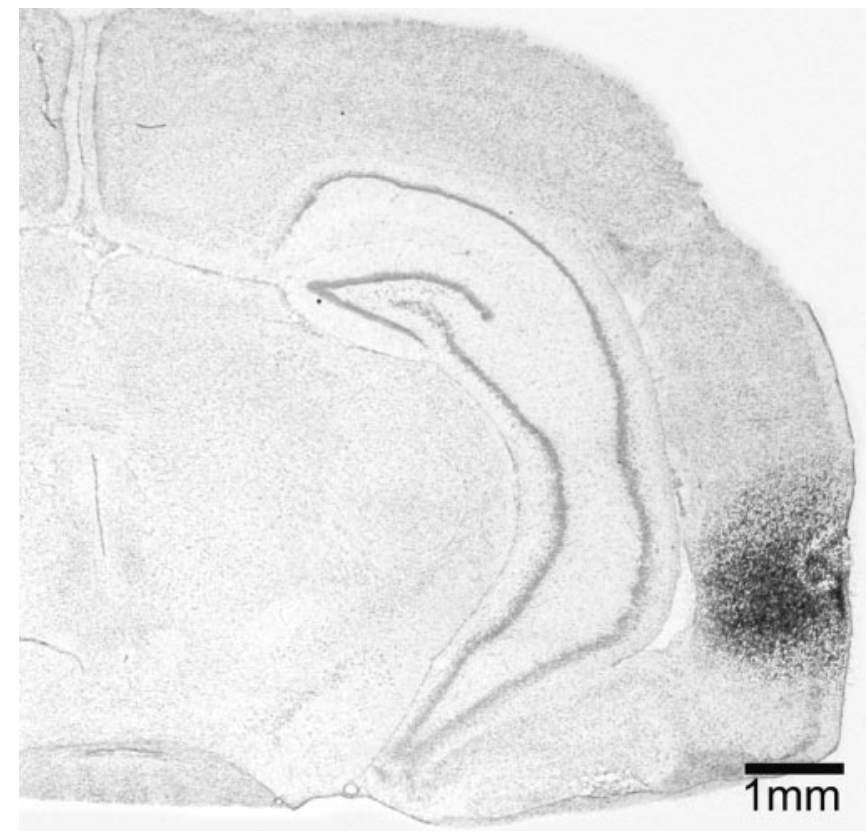

Figure 2. A representative section from the brain of a rat infused with $\left[{ }^{14} \mathrm{C}\right]$-lidocaine showing the typical area of diffusion within PRh. The radiolabeled image from the film has been superimposed onto the cresyl violet-stained section from the same animal. The section corresponds to $\sim 5.20 \mathrm{~mm}$ posterior to bregma (Paxinos and Watson, 1997).

$19.58 \mathrm{sec}$; pre-choice, saline $=117.66 \pm 23.39 \mathrm{sec}$, lidocaine $=$ $110.78 \pm 18.12 \mathrm{sec}$.

Object exploration during choice phase. There was no difference between groups in the total amount of time spent exploring the objects $\left(F_{(2,18)}=1.21\right)$, nor was the effect of drug $(F<1)$ or the group by drug interaction significant $\left(F_{(2,18)}=3.38\right)$. The mean exploration times $( \pm$ SEM $)$ were as follows: pre-sample, saline $=$ $13.99 \pm 1.48 \mathrm{sec}$, lidocaine $=12.74 \pm 1.66 \mathrm{sec}$; post-sample, saline $=13.30 \pm 1.71 \mathrm{sec}$, lidocaine $=18.82 \pm 1.92 \mathrm{sec}$; prechoice, saline $=15.98 \pm 1.59 \mathrm{sec}$, lidocaine $=12.95 \pm 1.77 \mathrm{sec}$.

Recognition during choice phase. Intra-PRh infusions of lidocaine were associated with a significant overall object recognition impairment; the performance of all three groups was substantially reduced on lidocaine trials when compared with performance on saline trials (Fig. 3). Analysis of the discrimination ratio yielded a significant effect of drug $\left(F_{(1,18)}=19.21 ; p<\right.$ $0.001)$; however, the group $(F<1)$ and interaction $(F<1)$ terms were not significant.

\section{Experimental discussion}

The results from experiment 1 demonstrate that PRh activity is required during the sample phase, the period immediately after the sample phase, and the choice phase for normal object recognition memory. These findings suggest a role for PRh in object encoding, storage, and retrieval. Experiment 2 a was conducted to examine in more detail the nature of the effects of pre-sample and pre-choice intra-PRh lidocaine infusions. Specifically, we wished to test the delay dependency of the effects observed in experiment 1 . Thus, rats were tested with pre-sample or pre-choice infusions with either $5 \mathrm{~min}$ or $180 \mathrm{~min}$ retention delays.

\section{Experiment 2a: encoding and retrieval in short- and long-term object recognition memory Histology \\ All animals had cannula placements as shown in Figure $1 a$ and therefore were included in the final analyses.}

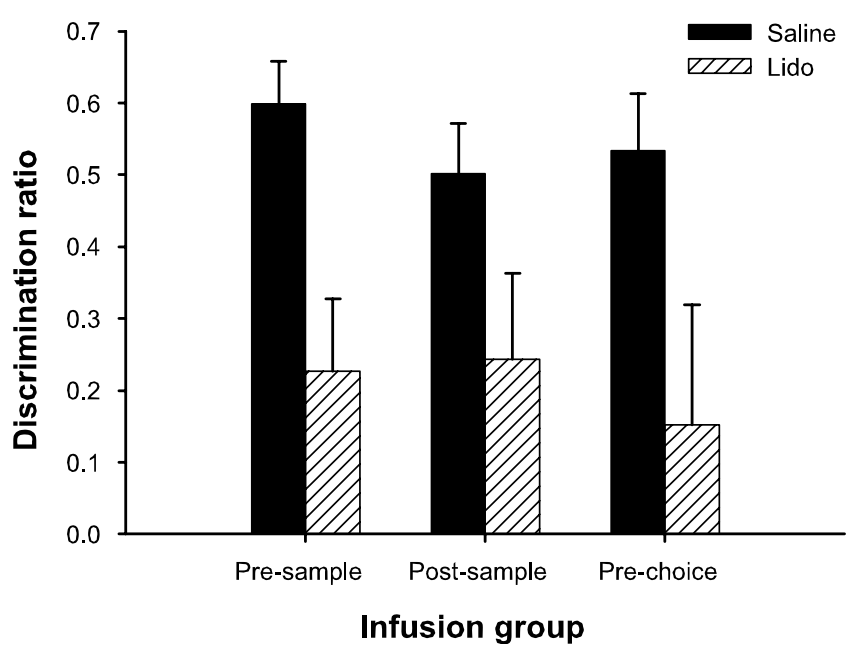

Figure 3. Spontaneous object recognition performance by animals in the pre-sample, postsample, and pre-choice infusions groups in experiment 1 on trials in which they received either saline or lidocaine (lido) infusions bilaterally into PRh. The retention delay was $20 \mathrm{~min}$. Data are presented as average discrimination ratio \pm SEM.

\section{Spontaneous object recognition}

Duration of sample phase. Analysis of the total time required to sample the objects for $25 \mathrm{sec}$ revealed no significant difference between the groups $\left(F_{(1,18)}=3.90\right)$, no significant effect of drug $(F<1)$, and no group by drug interaction $(F<1)$. The mean sample phase duration $( \pm$ SEM) for each group under each drug condition was as follows: pre-sample, saline $=143.40 \pm 8.91 \mathrm{sec}$, lidocaine $=142.37 \pm 13.46 \mathrm{sec}$; pre-choice, saline $=111.54 \pm$ $17.77 \mathrm{sec}$, lidocaine $=102.23 \pm 17.18 \mathrm{sec}$.

Object exploration during choice phase. There was no difference between groups in the total amount of time spent exploring the objects $\left(F_{(1,18)}=3.31\right)$, and both the effects of drug $(F<1)$ and the group by drug interaction $(F<1)$ failed to reach significance. The mean exploration times $( \pm$ SEM) were as follows: presample, saline $=17.11 \pm 1.54 \mathrm{sec}$, lidocaine $=15.64 \pm 0.86 \mathrm{sec}$; pre-choice, saline $=14.06 \pm 1.22 \mathrm{sec}$, lidocaine $=13.96 \pm 1.09$ sec.

Recognition during choice phase. Intra-PRh infusions of lidocaine substantially impaired both groups when compared with performance on saline trials, regardless of the length of the retention delay (Fig. 4). A three-way (group by drug by delay) ANOVA revealed significant effects of drug $\left(F_{(1,18)}=34.74 ; p<0.001\right)$ and delay $\left(F_{(1,18)}=17.93 ; p<0.001\right)$ but no significant group effect $\left(F_{(1,18)}=2.83\right)$. The group by drug $\left(F_{(1,18)}=2.41\right)$, group by delay $(F<1)$, drug by delay $(F<1)$, and group by drug by delay $(F<1)$ interaction terms all failed to reach significance.

\section{Experimental discussion}

The results from experiment $2 \mathrm{a}$ indicate that transient inactivation of PRh immediately before either the sample phase or choice phase disrupts object recognition with both $5 \mathrm{~min}$ and $180 \mathrm{~min}$ retention delays. These results extend the findings from the presample and pre-choice groups in experiment 1 and strongly suggest roles for PRh in both encoding and retrieval of object memory. Moreover, the delay independence of the deficits observed in experiment $2 \mathrm{a}$ is intriguing, because delay-dependent deficits after permanent $\mathrm{PRh}$ damage have been reported in humans (Buffalo et al., 1998), monkeys (Meunier et al., 1993; Buffalo et al., 1999, 2000), and rats (Ennaceur et al., 1996). Ennaceur et al. (1996), for example, reported that rats with neurotoxic PRh lesions are impaired with a $15 \mathrm{~min}$, but not a $1 \mathrm{~min}$, retention delay. 


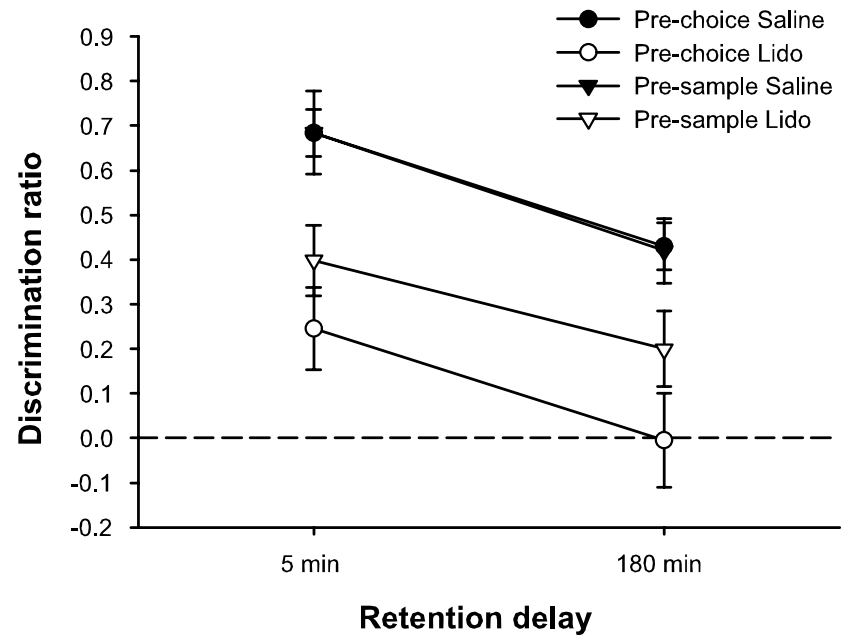

Figure 4. Spontaneous object recognition performance in experiment 2a by the pre-sample and pre-choice infusion groups at each of two different retention delays. Rats received either saline or lidocaine (lido) infusions bilaterally into PRh. Data are presented as average discrimination ratio $\pm \mathrm{SEM}$. Dashed line indicates chance performance (i.e., zero discrimination between familiar and novel objects).

The difference between these results and the present findings may highlight important differences between the effects of permanent and transient PRh lesions (see Discussion). It could also be argued, however, that we have not used a short enough retention delay to draw any firm conclusions about the delay dependency of the effects observed in experiment $2 a$. In experiment $2 b$, we sought to address this issue by testing the effects of pre-sample infusions with one extra delay: the minimum or shortest delay possible in the present version of the SOR task. Thus, in addition to the 5 and 180 min delays used in experiment $2 \mathrm{a}$, we added a minimum delay condition in which the retention delay duration was only as long as that required to change the objects between the sample and choice phases $(\sim 30 \mathrm{sec})$. There was no pre-choice infusion condition in experiment $2 \mathrm{~b}$, because this was not possible with the minimum delay.

\section{Experiment 2b: further encoding deficits in short- and long- term object recognition memory \\ Histology}

All animals had cannula placements as shown in Figure $1 a$ and therefore were included in the final analyses.

\section{Spontaneous object recognition}

Duration of sample phase. There was no significant difference between saline and lidocaine trials in the time required for rats to sample the objects for $25 \mathrm{sec}$, as revealed by a paired-samples $t$ test $(t<1)$. The mean sample phase duration $( \pm$ SEM) under each drug condition was as follows: saline $=132.21 \pm 10.22 \mathrm{sec}$, lidocaine $=133.43 \pm 11.42 \mathrm{sec}$.

Object exploration during choice phase. Lidocaine infusions did not affect the absolute time spent exploring the objects in the choice phase, as indicated by a paired-samples $t$ test $(t<1)$. The mean exploration times $( \pm \mathrm{SEM})$ were as follows: saline $=$ $17.29 \pm 3.33 \mathrm{sec}$, lidocaine $=16.49 \pm 2.96 \mathrm{sec}$.

Recognition during choice phase. Pre-sample intra-PRh infusions of lidocaine disrupted object recognition memory compared with saline infusions, regardless of the length of the retention delay (Fig. 5). Analysis of the discrimination ratio yielded significant effects of drug $\left(F_{(1,20)}=39.6 ; p<0.001\right)$ and delay

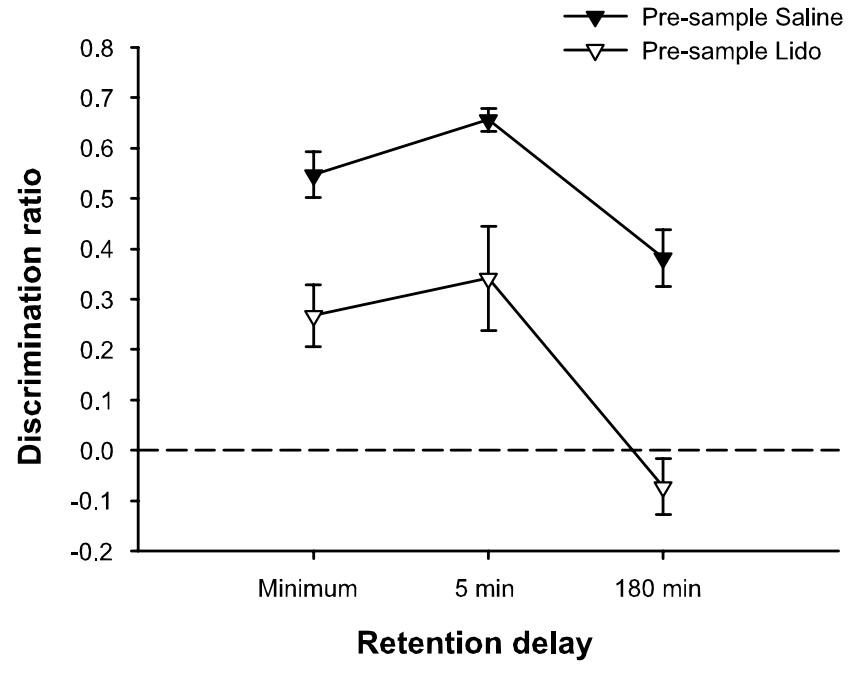

Figure 5. Spontaneous object recognition performance of animals in experiment $2 \mathrm{~b}$ at each of three different retention delays. Rats were infused before the sample phase with either saline or lidocaine (lido). Data are presented as average discrimination ratio \pm SEM. Dashed line indicates chance performance (i.e., zero discrimination between familiar and novel objects).

$\left(F_{(2,20)}=16.63 ; p<0.001\right)$, but the interaction term was not significant $\left(F_{(2,20)}=1.49\right)$.

\section{Experimental discussion}

These results replicate the delay-independent impairment observed in the pre-sample group in experiment $2 \mathrm{a}$ and extend this finding to show a deficit of similar magnitude with pre-sample infusions when the delay is as short as possible in the SOR task. The retention delay length in the minimum delay condition was never $>30 \mathrm{sec}$. Despite this very short retention delay, rats were impaired to the same degree in the minimum condition as they were in the 5 and 180 min conditions. The demonstration of impairment in object recognition at short delays in rats with transient pre-sample PRh lesions further suggests an important role for PRh in encoding of the object trace.

The findings of experiments $2 \mathrm{a}$ and $2 \mathrm{~b}$ indicate via replications that PRh activity is critical for normal object recognition during both sample and choice phases. In experiment 1 , it was also shown that PRh activity is necessary immediately postsample, when no objects are present. In experiments $3 \mathrm{a}$ and $3 \mathrm{~b}$, we explored further the nature of the deficit caused by postsample lidocaine infusions in experiment 1 by asking whether this impairment might reflect a requirement for PRh activity within a sensitive period after the sample phase to consolidate the object trace. We did this by infusing lidocaine or saline into PRh at different time points after the sample phase.

\section{Experiment 3a: consolidation of object recognition memory Histology}

One rat was excluded from the immediate infusion group because of cannula placements that were judged to be too lateral. All other animals had infusion needle tips located as shown in Figure $1 a$ and therefore were included in the final analyses.

\section{Spontaneous object recognition}

Duration of sample phase. Analysis of the sample phase duration revealed no significant difference between the groups $(F<1)$, no significant effect of drug $(F<1)$, and no group by drug interaction $(F<1)$. The mean sample phase duration ( \pm SEM) for each group under each drug condition was as follows: immediate, saline $=164.74 \pm 23.57 \mathrm{sec}$, lidocaine $=155.57 \pm 30.16 \mathrm{sec} ; 20$ 


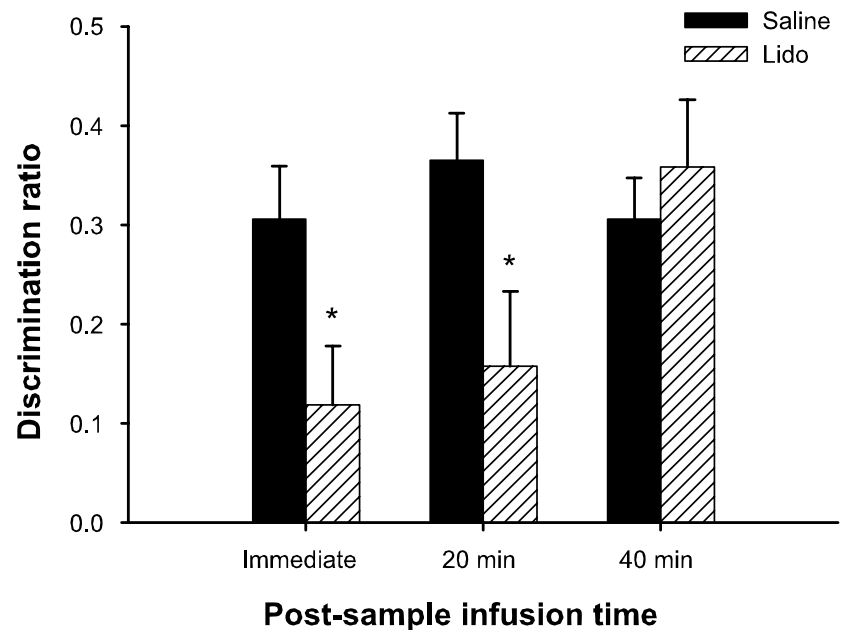

Figure 6. Spontaneous object recognition performance of the immediate, $20 \mathrm{~min}$, and 40 min post-sample infusion groups in experiment $3 \mathrm{a}$ on trials in which they received either saline or lidocaine (lido) infusions bilaterally into PRh. The retention delay was $60 \mathrm{~min}$. Data are presented as average discrimination ratio \pm SEM. ${ }^{*} p<0.05$, saline versus lidocaine.

min, saline $=150.03 \pm 19.55 \mathrm{sec}$, lidocaine $=157.42 \pm 22.39$ $\mathrm{sec} ; 40 \mathrm{~min}$, saline $=132.23 \pm 20.30 \mathrm{sec}$, lidocaine $=130.78 \pm$ $18.48 \mathrm{sec}$.

Object exploration during choice phase. There was no difference between groups in the total amount of time spent exploring the objects $(F<1)$, nor was the effect of drug $(F<1)$ or the group by drug interaction $(F<1)$ significant. The mean exploration time $( \pm \mathrm{SEM})$ for each group under each drug condition was as follows: immediate, saline $=16.26 \pm 2.04 \mathrm{sec}$, lidocaine $=16.20 \pm 1.64 \mathrm{sec}$; $20 \mathrm{~min}$, saline $=16.77 \pm 1.72 \mathrm{sec}$, lidocaine $=15.98 \pm 2.71 \mathrm{sec} ; 40$ min, saline $=17.11 \pm 2.14 \mathrm{sec}$, lidocaine $=15.39 \pm 1.66 \mathrm{sec}$.

Recognition during choice phase. Intra-PRh infusions of lidocaine impaired subsequent object recognition memory when the infusions were performed immediately and $20 \mathrm{~min}$, but not 40 min, after the sample phase (Fig. 6). Analysis of the discrimination ratio yielded a significant effect of drug $\left(F_{(1,23)}=8.92 ; p<\right.$ $0.01)$ and a significant drug by group interaction $\left(F_{(2,23)}=4.88\right.$; $p=0.02)$. The main effect of group was not significant $\left(F_{(2,23)}=\right.$ 1.83). Separate paired-samples $t$ tests were conducted to compare performance within each infusion group on saline and lidocaine trials. The results of these tests indicated significant differences between saline and lidocaine trial performance for the immediate $\left(t_{(7)}=2.87 ; p<0.05\right)$ and $20 \min \left(t_{(8)}=2.73, p<0.05\right)$ groups but not the $40 \mathrm{~min}$ infusion group $(t<1)$.

\section{Experimental discussion}

The results from experiment 3 a support the hypothesis that PRh activity is required for a sensitive period after stimulus encoding before the object memory trace becomes consolidated. Intra-PRh lidocaine infusions given immediately after the sample phase disrupted subsequent object recognition performance, replicating the effect observed in the post-sample group from experiment 1 . Interestingly, a similar impairment was seen when the lidocaine infusions were administered $20 \mathrm{~min}$ after the end of the sample phase. Object recognition was unimpaired, however, when rats were infused $40 \mathrm{~min}$ after the sample phase. This pattern of results suggests that ongoing neural activity in $\mathrm{PRh}$ is required to maintain the memory trace for a period of somewhere between 20 and 40 min post-sample. At some point between 20 and 40 min after encoding, the trace may become sufficiently strengthened by consolidatory mechanisms such that disruption of PRh activity with lidocaine is no longer detrimental to subsequent object recognition. In experiment $3 b$, we attempted to replicate and extend this pattern of results to test whether PRh inactivation within 20 min after the sample does indeed reliably disrupt subsequent object recognition performance and to show that inactivation beyond the 40 min time point tested in experiment $3 \mathrm{a}$ would not impair object recognition, providing the infusions are administered in sufficient time to allow the effects of lidocaine to dissipate before the choice phase. Thus, rats were tested after post-sample infusions at one of four time points within the retention delay: immediately, 20,60, or $80 \mathrm{~min}$ after the end of the sample phase. The retention delay was $120 \mathrm{~min}$.

\section{Experiment 3b: consolidation of object recognition memory: replication and extension \\ Histology}

All rats had cannula placements as shown in Figure $1 a$ and therefore were included in the final analyses.

\section{Spontaneous object recognition}

Duration of sample phase. Analysis of the total time in the apparatus during the sample phase revealed no significant difference between the groups $(F<1)$, no significant effect of drug $(F<1)$, and no group by drug interaction $(F<1)$. The mean sample phase duration $( \pm$ SEM $)$ for each group under each drug condition was as follows: immediate, saline $=72.25 \pm 8.83 \mathrm{sec}$, lidocaine $=73.66 \pm 16.01 \mathrm{sec}$; $20 \mathrm{~min}$, saline $=77.05 \pm 7.17 \mathrm{sec}$, lidocaine $=90.22 \pm 26.17 \mathrm{sec} ; 60$ min, saline $=71.59 \pm 7.86 \mathrm{sec}$, lidocaine $=76.02 \pm 7.29 \mathrm{sec} ; 80 \mathrm{~min}$, saline $=83.65 \pm 11.36 \mathrm{sec}$, lidocaine $=73.82 \pm 10.42 \mathrm{sec}$.

Object exploration during choice phase. There was no difference between groups in the total amount of time spent exploring the objects $(F<1)$, nor was the effect of drug $(F<1)$ or the group by drug interaction $(F<1)$ significant. The mean exploration time $( \pm \mathrm{SEM})$ for each group under each drug condition was as follows: immediate, saline $=16.97 \pm 1.53 \mathrm{sec}$, lidocaine $=15.49 \pm 1.61 \mathrm{sec}$; $20 \mathrm{~min}$, saline $=14.25 \pm 2.80 \mathrm{sec}$, lidocaine $=16.16 \pm 1.97 \mathrm{sec} ; 60$ min, saline $=18.22 \pm 1.50 \mathrm{sec}$, lidocaine $=15.81 \pm 1.99 \mathrm{sec} ; 80 \mathrm{~min}$, saline $=16.53 \pm 0.97 \mathrm{sec}$, lidocaine $=15.94 \pm 1.70 \mathrm{sec}$.

Recognition during choice phase. Intra-PRh infusions of lidocaine impaired subsequent object recognition memory when the infusions were performed immediately and $20 \mathrm{~min}$ after, but not 60 or $80 \mathrm{~min}$ after, the sample phase (Fig. 7). Analysis of the discrimination ratio indicated a significant effect of $\operatorname{drug}\left(F_{(1,24)}=\right.$ 15.19; $p<0.01)$ and a significant drug by group interaction $\left(F_{(3,24)}=5.28 ; p<0.01\right)$. The main effect of group was not significant $\left(F_{(3,24)}=2.56\right)$. Separate paired-samples $t$ tests comparing performance within each infusion group on saline and lidocaine trials revealed significant differences between saline and lidocaine trial performance for the immediate $\left(t_{(6)}=6.05 ; p<\right.$ $0.01)$ and $20 \mathrm{~min}\left(t_{(6)}=3.16 ; p<0.05\right)$ groups but not the $60 \mathrm{~min}$ $(t<1)$ or $80 \mathrm{~min}(t<1)$ infusion groups.

\section{Experimental discussion}

As in experiment 3a, PRh inactivation immediately and 20 min after encoding disrupted object recognition in a subsequent choice test. Choice performance was unimpaired, however, by intra-PRh infusions of lidocaine when the infusions were given 60 or $80 \mathrm{~min}$ after the sample phase. Thus, it seems that uninterrupted $\mathrm{PRh}$ activity is required for maintenance of the object trace for a period of $20-40 \mathrm{~min}$, after which the trace is sufficiently robust to resist disruption of PRh activity. The apparently necessary ongoing PRh neural activity during the early postsample period may facilitate activity-dependent synaptic plastic- 


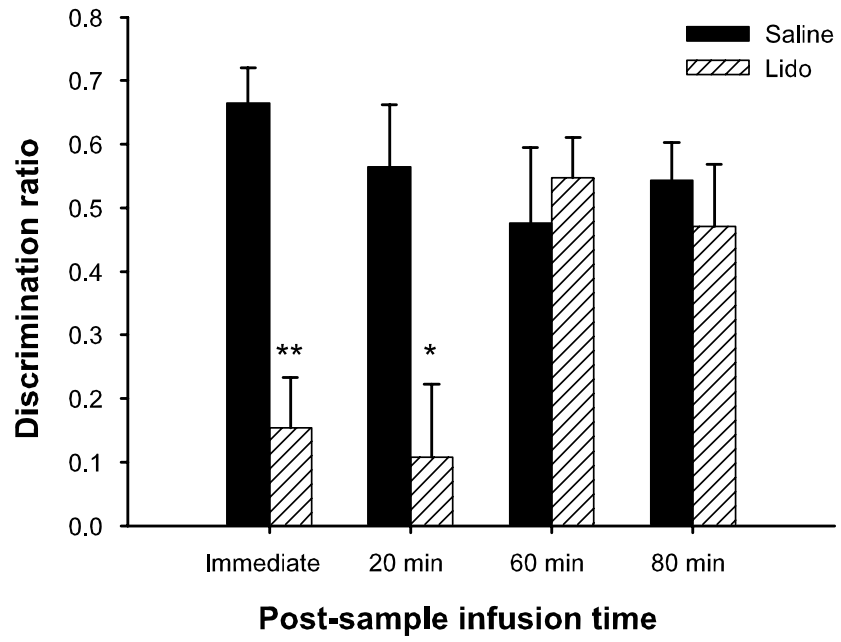

Figure 7. Spontaneous object recognition performance of the immediate and 20,60, and 80 min post-sample infusion groups in experiment $3 \mathrm{~b}$ on trials in which they received either saline or lidocaine (lido) infusions bilaterally into PRh. The retention delay was $120 \mathrm{~min}$. Data are presented as average discrimination ratio \pm SEM. ${ }^{*} p<0.05$, saline versus lidocaine; ${ }^{* *} p<$ 0.01 , saline versus lidocaine.

ity processes within $\mathrm{PRh}$ required for longer-term storage of the object memory trace.

\section{Discussion}

The present study provides evidence for the involvement of PRh in three distinct stages of object recognition memory. Transient inactivation of PRh immediately before the sample or choice phases impaired object recognition with short and long retention delays, and inactivation within the delay period disrupted object recognition when infusions were performed immediately or 20 min after the sample phase, but not later. Although two recent studies have examined the effects of transient PRh lesions on object recognition (Barnes et al., 2000; Hannesson et al., 2004), to our knowledge the present study is the first to examine comprehensively the role of PRh in encoding, retrieval, and consolidation of object recognition memory.

\section{Encoding}

Infusions of lidocaine into PRh immediately before the sample phase significantly impaired object recognition. This effect was seen with a very short (minimum) retention delay, as well as with delays of 5, 20, and $180 \mathrm{~min}$. These results suggest a significant role for PRh in the initial encoding of the object trace. It is possible, however, that lidocaine-induced inactivation continued for some time after the end of the sample phase and that post-sample inactivation may have contributed somewhat to the deficits observed in pre-sample lidocaine conditions. Importantly, however, the degree of impairment after pre-sample and post-sample infusions was roughly the same, yet the level of PRh inactivation at the end of the sample phase on a pre-sample trial would be expected to be lower than the inactivation caused by an immediate post-sample infusion. It is thus unlikely that the behavioral effects of pre-sample lidocaine can be attributed solely to postsample neural inactivation on pre-sample infusion trials.

Moreover, the idea that intra-PRh lidocaine could disrupt encoding is consistent with recent studies implicating PRh in object identification and perceptual representation (Buckley and Gaffan, 1998a,b; Murray and Bussey, 1999; Buckley et al., 2001; Bussey and Saksida, 2002; Bussey et al., 2002a,b), and it is well estab- lished that PRh lesions can disrupt visual discrimination as well as object recognition (Buckley and Gaffan, 1997; Buckley et al., 2001; Eacott et al., 2001; Bussey et al., 2003). Such findings suggest that PRh houses complex representations of objects. Therefore, the effect of pre-sample intra-PRh lidocaine in the present study may reflect impairment in the encoding of the perceptual representation of the object (Dudai, 2002). This may be the same representation that is remembered across a delay and used for subsequent memory retrieval. Alternatively, the effect of intraPRh lidocaine may have been caused by the disruption of any kind of useable visual representation. This seems unlikely, however, because PRh lesions do not tend to disrupt simple visual discrimination (Buckley et al., 2001; Bussey et al., 2003). Rather, impairment in visual discrimination emerges only when the nature of the to-be-discriminated stimuli is manipulated in such a way as to encourage the use of complex representations (Buckley et al., 2001; Bussey and Saksida, 2002; Bussey et al., 2002b, 2003). This issue could be addressed more specifically by examining the effects of PRh inactivation on simple versus complex visual discrimination tasks.

\section{Retrieval}

Inactivation of PRh just before the choice phase disrupted object recognition with retention delays of 5, 20, and $180 \mathrm{~min}$. The exact process implicated in this impairment is unclear. The effect of pre-choice lidocaine may indicate a specific role for $\mathrm{PRh}$ in the retrieval of the object trace to facilitate judgment of its previous presentation. The impairment could, however, also represent a deficit related to object identification and the visual discrimination of the two objects in the choice phase. Whatever the mechanism, the present results indicate a critical role for PRh at the retrieval stage of object recognition memory, consistent with previous transient inactivation (Hannesson et al., 2004) and lesion (Mumby et al., 2002) studies and suggest that PRh is important for the "actualization" or expression of remembered object information (Dudai, 2002).

\section{Consolidation}

The post-sample infusion conditions in experiments $1,3 \mathrm{a}$, and $3 \mathrm{~b}$ revealed a role for $\mathrm{PRh}$ in the consolidation of object recognition memory. Many types of memory remain labile and sensitive to disruption shortly after acquisition and stabilize progressively over time (McGaugh, 2000). Inactivation of PRh immediately or up to $20 \mathrm{~min}$ after the sample phase disrupted object recognition memory, whereas inactivation at 40,60 , or 80 min post-sample had no such effect. These results suggest an important function for PRh neural activity in the maintenance of the object trace during the delay. The fact that subsequent performance was unaffected by inactivation of $\mathrm{PRh}$ at $40 \mathrm{~min}$ post-sample and beyond suggests that the trace becomes resistant to disruption over a period of 20-40 min after sample presentation. Once this period of consolidation has ended, neural activity in PRh is no longer required, possibly because sufficient time has passed for the establishment of cellular and molecular processes involved in synaptic changes required for longer-term retention of the trace (Goelet et al., 1986; Dudai, 1996; Martin et al., 2000). Consistent with this hypothesis, we have recently found that immediate, but not $40 \mathrm{~min}$, post-sample blockade of either AMPA or NMDA receptors in $\mathrm{PRh}$ abolishes object recognition memory with a retention delay of $3 \mathrm{hr}$ (B. D. Winters and T. J. Bussey, unpublished observations). These glutamate receptors are crucial for synaptic plasticity processes such as long-term potentiation (LTP) and long-term depression (LTD) (Bliss and Collingridge, 
1993; Ziakopoulos et al., 1999; Cho et al., 2000; Martin et al., 2000). Accordingly, the effects of post-sample intra-PRh lidocaine in the present study may reflect interference with neuronal firing required for the establishment of activity- and NMDA receptor-dependent synaptic plasticity within PRh. Indeed, there is evidence that NMDA receptor-dependent LTP and LTD both occur in PRh (Bilkey, 1996; Ziakopoulos et al., 1999), and these provide likely candidates for the neural substrate of object recognition memory (Brown and Bashir, 2002).

To our knowledge, the electrophysiological effects of lidocaine within PRh have not been studied. It has been reported, however, that maintenance of activity-dependent synaptic plasticity in visual cortex in vitro requires neural activity, and blockade of action potentials with lidocaine after tetanus can completely abolish certain forms of LTP (Liu et al., 2004). Similarly, the present pattern of results suggests that initial encoding of the object trace in PRh does not ensure the completion of cellular and molecular changes required for long-term storage. Rather, the trace gradually transfers from a labile state, which requires ongoing PRh neural activity, to a more resistant memory over a period of 20-40 min after the sample phase.

Some final notes about the inactivation procedure are warranted. To assess the diffusion of lidocaine in the cortex, we infused animals with radiolabeled lidocaine and analyzed the diffusion extent using autoradiography. Fortuitously, the diffusion extended the full length of $\mathrm{PRh}$, without encroaching dorsally into area TE or ventrally into entorhinal cortex. This asymmetric spread may have been caused by several factors, including the presence, dorsally, of the guide cannula, or the closeness of the infusion site to the rhinal sulcus.

Although other agents such as muscimol have been used to induce transient inactivation in the brain, we chose the sodium channel blocker lidocaine primarily because of its short-lasting effects. Lidocaine, however, can disrupt fibers of passage, and this may have contributed to the behavioral effects reported in this study. This seems unlikely, however, because we have recently observed almost identical object recognition deficits after intra$\mathrm{PRh}$ infusions of the AMPA receptor antagonist CNQX, which does not affect fibers of passage (Winters and Bussey, unpublished observations).

The effects of both pre-sample and pre-choice lidocaine were evident at very short delays, consistent with a role for PRh in encoding perceptual representations. We cannot be certain, however, that these treatments would have the same effects with even shorter retention delays. Nonetheless, it is interesting that the minimum delay condition in the present study was much shorter than that usually required to bring out impairment in rats with permanent PRh lesions (Ennaceur et al., 1996). Permanent lesions may allow for recovery of function through compensatory changes. By reducing the likelihood of such adaptive changes, transient lesions might be expected to produce greater, and perhaps more informative, impairments.

\section{Conclusion}

The present results suggest involvement of PRh neural activity in the encoding and retrieval stages of object recognition memory. In addition, PRh neural activity is required for maintenance of the representation during the consolidation period that follows initial encoding of the object trace. The pattern of object recognition deficits resulting from $\mathrm{PRh}$ inactivation is similar to that seen in spatial memory with reversible AMPA-kainate receptor blockade in the hippocampus (Riedel et al., 1999). The similarity between the present results and those of Riedel et al. (1999) ex- amining a different type of memory in a different region of the brain may indicate a general characteristic of information processing whereby those brain structures positioned anatomically to receive certain types of information are involved at all stages of perceptual and mnemonic processing of that information (Gaffan, 2002).

\section{References}

Barnes SJ, Floresco SB, Kornecook TJ, Pinel JP (2000) Reversible lesions of the rhinal cortex produce delayed non-matching-to-sample deficits in rats. NeuroReport 11:351-354.

Bartolini L, Casamenti F, Pepeu G (1996) Aniracetam restores object recognition impaired by age, scopolamine, and nucleus basalis lesions. Pharmacol Biochem Behav 53:277-283.

Bilkey DK (1996) Long-term potentiation in the in vitro perirhinal cortex displays associative properties. Brain Res 733:297-300.

Bliss TV, Collingridge GL (1993) A synaptic model of memory: long-term potentiation in the hippocampus. Nature 361:31-39.

Boeijinga PH, Mulder AB, Pennartz CM, Manshanden I, Lopes da Silva FH (1993) Responses of the nucleus accumbens following fornix/fimbria stimulation in the rat. Identification and long-term potentiation of mono- and polysynaptic pathways. Neuroscience 53:1049-1058.

Bozon B, Kelly A, Josselyn SA, Silva AJ, Davis S, Laroche S (2003) MAPK, CREB and zif268 are all required for the consolidation of recognition memory. Philos Trans R Soc Lond B Biol Sci 358:805-814.

Brown MW, Bashir ZI (2002) Evidence concerning how neurons of the perirhinal cortex may effect familiarity discrimination. Philos Trans R Soc Lond B Biol Sci 357:1083-1095.

Buckley MJ, Gaffan D (1997) Impairment of visual object-discrimination learning after perirhinal cortex ablation. Behav Neurosci 111:467-475.

Buckley MJ, Gaffan D (1998a) Perirhinal cortex ablation impairs visual object identification. J Neurosci 18:2268-2275.

Buckley MJ, Gaffan D (1998b) Learning and transfer of object-reward associations and the role of the perirhinal cortex. Behav Neurosci 112:15-23.

Buckley MJ, Booth MC, Rolls ET, Gaffan D (2001) Selective perceptual impairments after perirhinal cortex ablation. J Neurosci 21:9824-9836.

Buffalo EA, Reber PJ, Squire LR (1998) The human perirhinal cortex and recognition memory. Hippocampus 8:330-339.

Buffalo EA, Ramus SJ, Clark RE, Teng E, Squire LR, Zola SM (1999) Dissociation between the effects of damage to perirhinal cortex and area TE. Learn Mem 6:572-599.

Buffalo EA, Ramus SJ, Squire LR, Zola SM (2000) Perception and recognition memory in monkeys following lesions of area TE and perirhinal cortex. Learn Mem 7:375-382.

Burwell RD (2001) Borders and cytoarchitecture of the perirhinal and postrhinal cortices in the rat. J Comp Neurol 437:17-41.

Bussey TJ, Saksida LM (2002) The organization of visual object representations: a connectionist model of effects of lesions in perirhinal cortex. Eur J Neurosci 15:355-364.

Bussey TJ, Muir JL, Aggleton JP (1999) Functionally dissociating aspects of event memory: the effects of combined perirhinal and postrhinal cortex lesions on object and place memory in the rat. J Neurosci 19:495-502.

Bussey TJ, Duck J, Muir JL, Aggleton JP (2000) Distinct patterns of behavioural impairments resulting from fornix transection or neurotoxic lesions of the perirhinal and postrhinal cortices in the rat. Behav Brain Res 111:187-202.

Bussey TJ, Saksida LM, Murray EA (2002a) The role of perirhinal cortex in memory and perception: conjunctive representations for object identification. In: The parahippocampal region: organization and role in cognitive functions (Witter MP, Wouterlood FG, eds), pp 239-254. Oxford: Oxford UP.

Bussey TJ, Saksida LM, Murray EA (2002b) Perirhinal cortex resolves feature ambiguity in complex visual discriminations. Eur J Neurosci 15:365-374.

Bussey TJ, Saksida LM, Murray EA (2003) Impairments in visual discrimination after perirhinal cortex lesions: testing "declarative" vs. "perceptualmnemonic" views of perirhinal cortex function. Eur J Neurosci 17:649-660.

Cho K, Kemp N, Noel J, Aggleton JP, Brown MW, Bashir ZI (2000) A new form of long-term depression in the perirhinal cortex. Nat Neurosci 3:150-156.

Clark RE, Zola SM, Squire LR (2000) Impaired recognition memory in rats after damage to the hippocampus. J Neurosci 20:8853-8860. 
Dudai Y (1996) Consolidation: fragility on the road to the engram. Neuron $17: 367-370$.

Dudai Y (2002) Memory from A to Z: keywords, concepts, and beyond. Oxford: Oxford UP.

Duzel E, Habib R, Rotte M, Guderian S, Tulving E, Heinze HJ (2003) Human hippocampal and parahippocampal activity during visual associative recognition memory for spatial and nonspatial stimulus configurations. J Neurosci 23:9439-9444.

Eacott MJ, Machin PE, Gaffan EA (2001) Elemental and configural visual discrimination learning following lesions to perirhinal cortex in the rat. Behav Brain Res 124:55-70.

Elliott R, Dolan RJ (1998) The neural response in short-term visual recognition memory for perceptual conjunctions. NeuroImage 7:14-22.

Ennaceur A, Delacour J (1988) A new one-trial test for neurobiological studies of memory in rats. 1: Behavioral data. Behav Brain Res 31:47-59.

Ennaceur A, Neave N, Aggleton JP (1996) Neurotoxic lesions of the perirhinal cortex do not mimic the behavioural effects of fornix transection in the rat. Behav Brain Res 80:9-25.

Floresco SB, Seamans JK, Phillips AG (1996) Differential effects of lidocaine infusions into the ventral CA1/subiculum or the nucleus accumbens on the acquisition and retention of spatial information. Behav Brain Res 81:163-171.

Forwood SE, Winters BD, Bussey TJ (2005) Hippocampal lesions that abolish spatial maze performance spare object recognition memory at delays of up to 48 hours. Hippocampus, in press.

Gaffan D (1994) Dissociated effects of perirhinal cortex ablation, fornix transection and amygdalectomy: evidence for multiple memory systems in the primate temporal lobe. Exp Brain Res 99:411-422.

Gaffan D (2002) Against memory systems. Philos Trans R Soc Lond B Biol Sci 357:1111-1121.

Goelet P, Castellucci VF, Schacher S, Kandel ER (1986) The long and the short of long-term memory-a molecular framework. Nature 322:419-422.

Hannesson DK, Howland JG, Phillips AG (2004) Interaction between perirhinal and medial prefrontal cortex is required for temporal order but not recognition memory for objects in rats. J Neurosci 24:4596-4604.

Liu HN, Kurotani T, Ren M, Yamada K, Yoshimura Y, Komatsu Y (2004) Presynaptic activity and $\mathrm{Ca}^{2+}$ entry are required for the maintenance of NMDA receptor-independent LTP at visual cortical excitatory synapses. J Neurophysiol 92:1077-1087.

Manns JR, Stark CE, Squire LR (2000) The visual paired-comparison task as a measure of declarative memory. Proc Natl Acad Sci USA 97:12375-12379.

Martin SJ, Grimwood PD, Morris RGM (2000) Synaptic plasticity and memory: an evaluation of the hypothesis. Annu Rev Neurosci 23:649-711.

McGaugh JL (2000) Memory-a century of consolidation. Science 287: 248-251.

McKee RD, Squire LR (1993) On the development of declarative memory. J Exp Psychol Learn Mem Cognit 19:397-404.

Meunier M, Bachevalier J, Mishkin M, Murray EA (1993) Effects on visual recognition of combined and separate ablations of the entorhinal and perirhinal cortex in rhesus monkeys. J Neurosci 13:5418-5432.

Mishkin M (1978) Memory in monkeys severely impaired by combined but not by separate removal of amygdala and hippocampus. Nature 273:297-298.
Mumby DG, Pinel JP (1994) Rhinal cortex lesions and object recognition in rats. Behav Neurosci 108:11-18

Mumby DG, Glenn MJ, Nesbitt C, Kyriazis DA (2002) Dissociation in retrograde memory for object discriminations and object recognition in rats with perirhinal cortex damage. Behav Brain Res 132:215-226.

Murray EA, Bussey TJ (1999) Perceptual-mnemonic functions of the perirhinal cortex. Trends Cogn Sci 3:142-151.

Murray EA, Mishkin M (1998) Object recognition and location memory in monkeys with excitotoxic lesions of the amygdala and hippocampus. J Neurosci 18:6568-6582.

Nemanic S, Alvarado MC, Bachevalier J (2004) The hippocampal/parahippocampal regions and recognition memory: insights from visual paired comparison versus object-delayed nonmatching in monkeys. J Neurosci 24:2013-2026.

Pascalis O, Bachevalier J (1999) Neonatal aspiration lesions of the hippocampal formation impair visual recognition memory when assessed by paired-comparison task but not by delayed nonmatching-to-sample task. Hippocampus 9:609-616.

Paxinos G, Watson C (1997) The rat brain in stereotaxic coordinates, Ed 3. London: Academic.

Pitsikas N, Rigamonti AE, Cella SG, Locatelli V, Sala M, Muller EE (2001) Effects of molsidomine on scopolamine-induced amnesia and hypermotility in the rat. Eur J Pharmacol 426:193-200.

Richmond J, Sowerby P, Colombo M, Hayne H (2004) The effect of familiarization time, retention interval, and context change on adults' performance in the visual paired-comparison task. Dev Psychobiol 44:146-155.

Riedel G, Micheau J, Lam AG, Roloff E, Martin SJ, Bridge H, Hoz L, Poeschel B, McCulloch J, Morris RGM (1999) Reversible neural inactivation reveals hippocampal participation in several memory processes. Nat Neurosci 2:898-905.

Seamans JK, Phillips AG (1994) Selective memory impairments produced by transient lidocaine-induced lesions of the nucleus accumbens in rats. Behav Neurosci 108:456-468.

Tang YP, Shimizu E, Dube GR, Rampon C, Kerchner GA, Zhuo M, Liu G, Tsien JZ (1999) Genetic enhancement of learning and memory in mice. Nature 401:63-69.

Winters BD, Forwood SE, Cowell RA, Saksida LM, Bussey TJ (2004) Double dissociation between the effects of peri-postrhinal cortex and hippocampal lesions on tests of object recognition and spatial memory: heterogeneity of function within the temporal lobe. J Neurosci 24:5901-5908.

Ziakopoulos Z, Tillett CW, Brown MW, Bashir ZI (1999) Input- and layerdependent synaptic plasticity in the rat perirhinal cortex in vitro. Neuroscience 92:459-472.

Zola-Morgan S, Squire LR (1985) Medial temporal lesions in monkeys impair memory on a variety of tasks sensitive to human amnesia. Behav Neurosci 99:22-34.

Zola-Morgan S, Squire LR, Amaral DG (1986) Human amnesia and the medial temporal region: enduring memory impairment following a bilateral lesion limited to field CA1 of the hippocampus. J Neurosci 6:2950-2967.

Zola-Morgan S, Squire LR, Amaral DG, Suzuki WA (1989) Lesions of perirhinal and parahippocampal cortex that spare the amygdala and hippocampal formation produce severe memory impairment. J Neurosci 9:4355-4370. 